\title{
Leitura contextual e o processamento metalinguístico: considerações teóricas ${ }^{1}$
}

\author{
Márcia Maria Peruzzi Elia da Mota² \\ Silvia Brilhante Guimarães \\ Universidade Federal de Juiz de Fora, Juiz de Fora-MG, Brasil
}

\begin{abstract}
Resumo: $\mathrm{O}$ ato de ler não é um processo simples, pois envolve uma série de operações cognitivas, principalmente operações metalinguísticas. Estudos que investigam o papel dessas habilidades, na leitura, demonstram que a capacidade de refletir sobre os sons da fala, a consciência fonológica, contribui para leitura de palavras isoladas e compreensão de texto, ajudando a decodificação. Já outros estudos apontam que as pistas sintáticas e semânticas presentes no contexto são um forte recurso para a leitura, principalmente em leitores com dificuldades, pois ajudam a criança a reconhecer as palavras sem mediação fonológica. Recentemente, modelos interativos sugerem que a leitura contextual envolve a mediação fonológica que interage com as informações sintático-semânticas. Este estudo discute as implicações teóricas dessa questão.
\end{abstract}

Palavras-chave: metalinguagem, consciência fonológica, leitura, compreensão da leitura.

\section{Contextual reading and metalinguistic processing: theorectical considerations}

\begin{abstract}
The act of reading is not a simple process, as it involves a series of cognitive operations, specially metalinguisticoperations. Studies that investigate the role that these abilities play in reading show that the capacity to reflect upon word's sounds, phonological awareness, contributes to reading single words and reading comprehension. Others point out that syntact and semantic cues from context are a powerful resource for aiding reading, especially for readers, that experience difficulties, as they help the child to recognize words without phonological mediation. Recently, interactive models suggest that contextual reading interact with syntactic/semantic information. This papers discuss this issue theoretical implications.
\end{abstract}

Keywords: metalanguage, phonological awareness, reading, reading comprehension.

\section{Lectura contextual y proceso metalingüístico: consideraciones teóricas}

Resumen: El acto de leer no es un proceso simple, por que engloba una clase de operaciones cognitivas, principalmente de las acciones metalingüísticas. Estudios que investigan el papel de estas habilidades en la lectura demuestran que la capacidad de reflecións sobre los sonidos de hablar, la consciencia fonológica, contribuye para lectura de palabras aislados y comprensión de textos, ayudando a decodificación. Ya otros estudios apuntan que las pistas semánticas e sintácticas del contexto, consciencia morfosintáctica, son un fuerte recurso para la lectura, principalmente en lectores, con dificultades, pues ayudan los niños reconocer las palabras sin mediación fonológica. Recientemente, modelos interactivos sugieren que la lectura contextual engloba la mediación fonológica que interactúa con las informaciones sintáctico/semántico. Ese artigo discute las implicaciones teóricas de la cuestión.

Palabras clave: metalenguaje, conciencia fonológica, lectura, compresión de lectura.

Nos últimos 30 anos, houve um aumento expressivo do número de estudos que investigaram a relação entre a consciência metalinguística e a alfabetização (Bowey, 2005; Bradley \& Bryant, 1983; Gombert, 1992; Plaza \& Cohen, 2003, 2004). Dentre as habilidades metalinguísticas, duas se destacam por contribuírem para o sucesso na alfabetização como precursores: a consciência fonológica e a consciência morfossintática.

Dentre as habilidades metalinguísticas, a mais estudada é a consciência fonológica que pode ser definida como a habilidade de refletir sobre os sons que compõem a fala (Cardoso-Martins, 1995). Estudos demonstram que

${ }^{1}$ Este texto foi revisado seguindo o Acordo Ortográfico da Língua Portuguesa (1990), em vigor a partir de $1^{\circ}$ de janeiro de 2009.

${ }^{2}$ Endereço para correspondência:

Márcia Maria Peruzzi Elia da Mota. Universidade Federal de Juiz de Fora. Instituto de Ciências Humanas e Letras, campus Universitário Martelos. CEP 36.036-330. Juiz de Fora-MG, Brasil.E-mail: mmotapsi@pesquisador.cnpq.br a consciência fonológica ajuda na alfabetização e que o treinamento dessa habilidade ajuda na remediação dos problemas de leitura (Bradley \& Bryant, 1985; Cuninghan, 1990; Goswami \& Bryant, 1990). Pesquisadores brasileiros também confirmam a importância do tema para a aquisição da língua escrita no português brasileiro (Capovilla \& Capovilla, 2000; Cardoso-Martins, 1995; Guimarães, 2003; Barrera \& Maluf, 2003; Santos, 1996).

A consciência fonológica está geralmente associada à decodificação de palavras, pois ajudaria a criança a aprender o princípio alfabético. O princípio é o de que, nas línguas alfabéticas, as letras (grafemas) correspondem aos sons (fonema) da fala. A aquisição desse princípio é de grande importância para que se possa aprender a ler e escrever nesse tipo de ortografia. Diversos estudos têm demonstrado, no entanto, que essa habilidade contribui para a compreensão de leitura, à medida que favorece a leitura contextual (Tunmer, 1992). Isso ocorre provavelmente porque a automatização da decodificação acelera o processo de reconhecimento das 
palavras no texto o que, por sua vez, libera espaço da memória para armazenamento do texto, facilitando o processo de compreensão (Stanovich, 1980).

A consciência fonológica não é a única habilidade a facilitar o processo de alfabetização. Evidências vêm sendo acumuladas que mostram o papel facilitador da consciência morfológica na aquisição da leitura e escrita (Carlisle, 1988, 1995, 1996, 2000; Deacon \& Kirby, 2004; Nagy, Berninger, \& Abbot, 2006). Consciência morfológica é a habilidade de refletir sobre os morfemas, menores unidades linguísticas que têm significado próprio (Carlisle, 1995). Alguns autores preferem o termo consciência morfossintática a consciência morfológica, porque o processamento morfológico envolve também o processamento de aspectos semânticos e sintáticos (Correa, 2009). Neste artigo será usado o termo morfossintaxe. Essa habilidade pode ajudar na alfabetização porque a ortografia de muitas palavras depende da morfologia. Palavras como "laranjeira", que têm ortografia ambígua, podem ser escritas de forma correta se soubermos sua origem: "laranja". Os significados delas podem ser igualmente inferidos na leitura, se o leitor souber o significado da palavra que as originou.

Mann (2000) aponta duas razões para a importância da consciência morfossintática para alfabetização. A primeira diz respeito à argumentação de que a escrita pode ser analisada em vários níveis, não só o fonológico. Um deles é o nível semiográfico que implica estabelecer como os grafemas representam os significados das palavras (Marec-Breton \& Gombert, 2004). Considerando que os morfemas são unidades de significado, pode-se hipotetizar que a consciência morfológica pode facilitar a aquisição do princípio semiográfico. A segunda razão refere-se mais especificamente à natureza da ortografia estudada. As línguas alfabéticas variam quanto ao grau de correspondência entre as letras e os sons. Algumas línguas são muito "transparentes", isto é possuem relações entre letra e som bem diretas e sem ambiguidade, e outras línguas são mais "opacas", apresentando muitas palavras que não obedecem às regras de correspondência entre letra e som ou que podem ter várias grafias para o mesmo som, o que chamamos de ambiguidade.

É importante ressaltar que a argumentação principal para a relação encontrada entre o processamento morfológico e a alfabetização vem de pesquisas realizadas em falantes do inglês, língua nativa de Mann (2000) e dos sujeitos por ela estudados. No inglês essas relações são mais opacas do que em ortografias como o finlandês, o português ou o espanhol. Muitas das irregularidades encontradas no inglês podem ser explicadas pela estrutura morfológica das palavras (Chomsky \& Halle, 1968; Sterling, 1992). Por exemplo, no inglês a palavra 'heal' que rima com 'il' e a palavra 'health' que rima com 'elf' têm a mesma origem semântica; por isso são escritas da mesma forma, embora sejam pronunciadas de forma diferente.

Nas línguas com ortografias mais regulares, o processamento morfológico pode não contribuir de forma significativa para aquisição e processamento da língua escrita, porque a maioria das palavras pode ser escrita aplicando-se o princípio alfabético. Mann (2000) supõe que as línguas alfabéticas mais regulares podem ser mais dependentes da estrutura fonológica das palavras do que da estrutura morfológica. Lehtonen e Bryant (2005) ressaltam que, embora este seja um argumento válido, a hipótese de que a consciência morfológica contribui para alfabetização, também nas ortografias regulares, é pertinente, argumentando que essa é uma questão teórica que precisa ser mais bem investigada. De fato, muitos estudos realizados no português vêm demonstrando uma associação entre a consciência morfológica e a língua escrita (Curvelo, Meireles, \& Correa, 1998; Mota \& Silva, 2007; Mota, Annibal, \& Lima, 2008; Queiroga, Lins, \& Pereira, 2006). No francês, Colé, Marec-Breton, Royer e Gombert (2003) evidenciaram um fator facilitador da consciência morfológica na leitura de palavras isoladas em crianças de primeira série.

Rego e Bryant (1993) argumentaram que diferentes aspectos da consciência metalinguística podem contribuir de maneira diversa para a leitura e escrita. $\mathrm{Na}$ escrita, as crianças vão dos sons da palavra para o processamento gráfico. Nesse caso, há pouca ou nenhuma ajuda do contexto. A habilidade mais importante seria, então, a consciência fonológica que ajuda na aquisição do princípio alfabético, na decodificação. $\mathrm{Na}$ leitura, informações visuais, contextuais e fonológicas já são apresentadas no texto. A habilidade de refletir sobre a estrutura sintática das sentenças, denominada frequentemente como consciência sintática, ajudaria a criança a utilizar pistas contextuais para ler.

A hipótese de Tunmer (1990, 1992) difere da de Rego e Bryant (1993). Para Tunmer, na leitura contextual pistas grafofonêmicas podem se utilizadas com informações contextuais para a criança ler. Segundo o autor, a consciência fonológica influenciaria diretamente o processo de decodificação e indiretamente o processo de compreensão, enquanto a consciência sintática afetaria ambos os processos, uma vez que as pistas sintático-semânticas podem ser usadas tanto para reconhecer palavras novas ou difíceis no texto quanto para monitorar a compreensão dele. Assim quando a criança não reconhece uma palavra em uma frase, ela pode usar a decodificação parcial da palavra associada ao contexto para reconhecer a palavra que falta e garantir a compreensão do texto. Na frase "Vovó toma o ca**". O contexto oferece a informação de que se necessita de um substantivo masculino (o artigo "o" define o gênero) que se refere a algo que possa ser bebido. Porém, a decodificação parcial da sílaba "ca" mostra que somente bebidas que começam com $/ \mathrm{ka} /$ podem ser utilizadas na frase. Nessa perspectiva, tanto a consciência fonológica como a morfossintática contribuiriam para a leitura contextual. $O$ contexto ajudaria na aquisição de novas regras de correspondência letra e som.

O uso de informações contextuais na leitura é conhecido como processo top down ("de cima para baixo"). Durante muito tempo se defendeu que a leitura deveria prescindir da 
decodificação e focar na utilização de pistas contextuais apenas. Essa visão fundamenta-se em trabalhos publicados na década de 1960 e 1970, sobretudo no trabalho de Goodman que foi republicado na década de 1980 (Goodman, 1982). Esse autor propôs que a leitura deveria ser entendida sob a ótica da Psicolinguística. Nessa abordagem a criança tem um rico repertório linguístico que seria ativado na leitura. Por exemplo, as crianças conhecem desde muito cedo as regras gramaticais de sua língua, se comunicam de forma adequada, entendem a fala e a leitura de textos lidos para elas. $\mathrm{Na}$ leitura, a criança utilizaria seus conhecimentos linguísticos para compreender os textos escritos. Esses conhecimentos linguísticos ajudariam a criança a adivinhar as palavras que aparecem no texto, sem necessidade de decodificar as palavras que precisam ser lidas.

Não depender da decodificação é um aspecto importante para defensores desta abordagem. Smith (1971) lembra que a decodificação de quem começa a ler é lenta, ocupando muito tempo da memória de trabalho da criança. A memória de trabalho tem um componente temporal. O tempo gasto para a decodificação da palavra faria as outras informações presentes no texto serem esquecidas, o que dificultaria a interpretação do mesmo, tornando a leitura maçante para a criança. Nesse caso, a leitura contextual seria fundamental, pois aceleraria o processo. Dependendo menos da decodificação, a compreensão do texto ficaria facilitada. As práticas de alfabetização oriundas dessa abordagem valorizam o trabalho com o texto em que se busca o seu sentido. O reconhecimento das palavras do texto, em geral, está associado à formação de um vocabulário visual.

No entanto, estudos posteriores aos de Goodman (1982) e Smith (1971) como os de Nicholson (1986, 1993), Tunmer (1990), Stanovich (1980) e Shankweiler e Fowler (2004), alguns já citados, demonstraram que, ao contrário do que Goodman e Smith pensavam, a decodificação eficiente é um importante aspecto da aquisição da leitura. A esse respeito, Ryder, Turnmer e Greaney (2008) verificaram se a instrução explícita sobre os aspectos fonêmicos e as habilidades de decodificação seriam uma forma eficaz na estratégia de intervenção com crianças com dificuldades na aprendizagem. Os resultados demonstraram que o grupo de intervenção apresentou um desempenho excepcional em relação ao grupo de controle, sobretudo nas medidas de consciência fonêmica, decodificação de pseudopalavras, reconhecimento de palavras livre do contexto e de compreensão de leitura. Posteriormente, no follow-up, as crianças não só mantiveram os efeitos positivos da aprendizagem, mas ampliaram suas capacidades para a leitura de palavras no contexto. No entanto, esse estudo apresenta limitações quanto à metodologia empregada, uma vez que os aspectos sintáticos não foram controlados.

Stanovich (1980) propõe um modelo "interativo compensatório". Ressalta que, embora as pistas contextuais sejam utilizadas, os bons leitores (aqueles com maior facilidade de compreender a leitura) são justamente aqueles que são decodificadores mais eficientes. Bons leitores não são tão dependentes do contexto, pois decodificam bem as palavras, já os leitores principiantes e maus leitores compensam suas deficiências, dependendo mais das pistas contextuais. Em outras palavras, o processo top down parece ser importante para o leitor principiante ou para crianças com dificuldades de leitura. Essas precisam se valer do contexto para auxiliálas na leitura, haja vista a dificuldade que apresentam para a decodificação das palavras.

Em conclusão, o modelo interativo compensatório sugere que os leitores eficientes parecem ter menos dependência do contexto e serem melhores na decodificação (Stanovich, 1980). Posteriormente, Nicholson (1986) apoia o modelo de Stanovich.

Rego e Bryant (1993) testaram a hipótese de que diferentes estratégias de leitura e escrita estão associadas a diferentes habilidades metalinguísticas em crianças inglesas. Verificaram que, embora a consciência fonológica e a sintática contribuíssem para uma porção significativa na variância em testes de leitura, no caso da escrita, somente a consciência fonológica contribuía de forma estatisticamente significativa.

Posteriormente, Rego (1995) investigou a contribuição da consciência sintática para a leitura de palavras isoladas e no contexto, entre crianças brasileiras. Em um estudo longitudinal com crianças pré-leitoras, a autora mediu a habilidade de as crianças corrigirem palavras desordenadas numa frase (tarefa de consciência sintática). Cerca de um ano mais tarde, mediu a habilidade de as crianças lerem pseudopalavras, palavras isoladas e palavras inseridas num contexto. Se a consciência sintática contribuísse para a decodificação na leitura, então os escores na tarefa de consciência sintática deveriam predizer os escores na tarefa de leitura de pseudopalavras.

Os resultados das correlações feitas entre as diversas medidas tomadas por Rego (1995) mostraram que a medida de consciência sintática correlacionou-se de forma estatisticamente significativa e positiva com a tarefa de leitura de palavras inseridas num contexto, mas não com a tarefa de leitura de palavras isoladas ou de palavras inventadas. $\mathrm{O}$ resultado da regressão múltipla, que avaliou a contribuição dos escores na tarefa de consciência sintática para a leitura contextual um ano depois, mostrou um resultado estatisticamente significativo, mesmo depois de serem controlados a idade e os escores na tarefa de memória de trabalho.

Rego (1995) concluiu que, em línguas alfabéticas, quando as crianças são ensinadas pelo método silábico como foi o caso das crianças estudadas por ela, a sensibilidade fonológica parece não ser relevante para a leitura contextual. Porém, se as crianças são ensinadas explicitamente a refletir sobre unidades de som das palavras, seria esperado que elas generalizassem esse conhecimento para outras unidades de som que compõem a palavra. Uma explicação alternativa para os resultados de Rego é a de que um viés metodológico ocorreu, haja vista que, em seu estudo, a autora utilizou apenas tarefas 
de aliteração como medida de consciência fonológica. A tarefa de aliteração pode não ter sido adequada para avaliar a consciência fonológica das crianças.

Assim sendo, há uma importante questão conceitual a ser discutida e mais bem investigada: Que habilidades metalinguísticas contribuem para leitura contextual - leitura de palavras no contexto de sentenças e textos?

Mota e Santos (2009) investigaram a relação entre a consciência fonológica e a leitura contextual em crianças de segundo e terceiro anos. A consciência fonológica foi medida por duas tarefas, sendo uma de rima e outra de aliteração. Embora as duas tarefas tenham apresentado correlações positivas e significativas com a leitura contextual, a tarefa de rima manteve sua contribuição depois de ter sido controlado o efeito da idade, o que não ocorreu com a aliteração.

Dessa forma, os resultados oferecem alguma evidência que parece confrontar a teoria de $\operatorname{Goodman}(1965,1967)$ que sugere que as práticas de ensino da leitura e da escrita não devem envolver o ensino das regras de correspondência entre letra e som.

\section{Considerações finais}

Diante das considerações apresentadas, conclui-se que o ato de ler não é um processo simples, pois envolve uma série de operações cognitivas, principalmente a utilização das habilidades metalinguísticas descritas anteriormente (consciência fonológica e morfossintática). Diversos estudiosos vêm destrinchando essas habilidades, apontando seus limites e avanços implicados no processo de leitura. No entanto, esta área necessita de maiores aprofundamentos, para que se possa traçar uma proposta mais consistente de desenvolvimento do processo de leitura acerca das habilidades metalinguísticas.

\section{Referências}

Bowey, J. (2005). Grammatical sensitivity: Its origins and potential contribution to early reading skill. Journal of Experimental Child Psychology, 90(4), 318-343.

Barrera, S. D., \& Maluf, M. R. (2003). Consciência metalinguística e alfabetização: Um estudo com crianças da primeira série do ensino fundamental. Psicologia: Reflexão e Crítica, 16(3), 491-502.

Bradley, L., \& Bryant, P. (1983). Categorizing sounds and learning to read: A causal connection. Nature, 301(5899), 419-421.

Bradley, L., \& Bryant, P. (1985). Children's reading problems. Oxford, UK: Basil Blackwell.

Capovilla, A., \& Capovilla, F. (2000). Efeitos do treino de consciência fonológica em crianças com baixo nível sócio-econômico. Psicologia: Reflexão e Crítica, 13(1), 7-24.

Cardoso-Martins, C. (1995). Consciência fonológica e alfabetização. Petrópolis, RJ: Vozes.
Carlisle, J. (1988). Knowledge of derivational morphology and spelling ability in fourth, six, and eight graders. Applied Psycholinguistics, 9(3), 247-266.

Carlisle, J. (1995). Morphological awareness and early reading achievement. In L. Feldman (Ed.), Morphological aspects of language processing (pp. 189-211). Hillsdale, IN: Lawrence Erlbaum Associates.

Carlisle, J. (1996). An exploratory study of morphological errors in children's written stories. Reading and Writing: An Interdisciplinary Journal, 8(1), 61-72.

Carlisle, J. (2000). Awareness of the structure and meaning of morphologically complex words: Impact on reading. Reading and Writing: An Interdisciplinary Journal, 12(3-4), 169-190.

Chomsky, N., \& Halle, M. (1968). The sound patterns of english. New York: Harper \& Row.

Colé, P., Marec-Breton, N., Royer, C., \& Gombert, J. E. (2003). Morphologie des mots et apprentissage de la lecture. Reeducation Orthophonic, 213(1), 57-60.

Correa, J. (2009). Habilidades metalinguísticas relacionadas à sintaxeeàmorfologia. In M. Mota(Org.), Desenvolvimento metalinguístico: Questões contemporâneas (pp. 55-76). São Paulo: Casa do Psicólogo.

Cuningham, A. (1990). Explicit versus implicit instruction in phonemic awareness. Journal of Experimental Child Psychology, 50(3), 429-444.

Curvelo, C., Meireles, E., \& Correa, J. (1998). O conhecimento ortográfico da criança no jogo da forca. Psicologia: Reflexão \& Crítica, 11(3), 467-480.

Deacon, S., \& Kirby, J. (2004). Morphological awareness: Just more phonological? The roles of morphological and phonological awareness in reading development. Applied Psycholinguistics, 25(2), 223-238.

Goodman, K. (1965). A linguistic study of cues and miscues in reading. Elementary English, 42, 639-643.

Goodman, K. (1967). Reading: A psycholinguistic guessing game. Journal of the Reading Specialist, 6(4), 126-135.

Goodman, K. (1982). Language and literacy: The selected writings of Kenneth S. Goodman. Boston, MA: Routledge and Kegan Paul.

Gombert, J. E. (1992). Metalinguistic development. Hertfordshire, England: Harverster Wheatsheaf.

Goswami, U., \& Bryant, P. (1990). Phonological skills and learning to read. London: Lawrence Erlbaun Associates.

Guimarães, S. R. K. (2003). Dificuldades no desenvolvimento da lectoescrita: O papel das habilidades metalinguísticas. Psicologia: Teoria e Pesquisa, 19(1), 33-45.

Lehtonen, A., \& Bryant, P. (2005). Active players or just passive bystanders? The role of morphemes in spelling development in a transparent orthography. Applied Psycholinguistics, 26(2), 137-155.

Mann, V. (2000). Introduction to special issue on morphology and the acquisition of alphabetic writing systems. Reading and Writing: An Interdisciplinary Journal, 12(3-4), 143147. 
Marec-Breton, N., \& Gombert, J. (2004). A dimensão morfológica nos principais modelos de aprendizagem da leitura. In M. R. Maluf (Org.), Psicologia educacional: Questões contemporâneas (pp. 105-122). São Paulo: Casa do Psicólogo.

Mota, M., \& Silva, K. (2007). Consciência morfológica e desenvolvimento ortográfico: Um estudo exploratório. Psicologia em Pesquisa, 1(2), 86-92.

Mota, M., Annibal, L., \& Lima, S. (2008). A morfologia derivacional contribui para a leitura e escrita no português? Psicologia: Reflexão e Crítica, 21(2), 311-318.

Mota, M., \& Santos, A. A. (2009). O papel da consciência fonológica na leitura contextual medida pelo Cloze. Estudos de Psicologia (Natal), 14(3), 207-212.

Nagy, W., Berninger, V., \& Abbot, R. (2006). Contributions of morphology beyond phonology to literacy outcome of upper elementary and middle-school students. Journal of Educational Psychology, 98(1), 134-147.

Nilcholson, T. (1986). Reading is not a guessing game the great debate revited. Reading Psychology: An International Quarterly, 7(3), 197-210.

Nicholson, T. (1993). The case against context. In G. Thompson, W. Tunmer, \& T. Nicholson (Eds.), Reading acquisition processes (pp. 91-103). Clevedon, UK: Multilingual Matters.

Plaza, M., \& Cohen, H. (2003). The interaction between phonological processing, syntactic awareness, and naming speed in the reading and spelling performance of first-grade children. Brain and Cognition, 53(2), 287292.

Plaza, M., \& Cohen, H. (2004). Predictive influence of phonological processing, morphological/syntactic skill, and naming speed on spelling performance. Brain and Cognition, 55(2), 368-373.

Queiroga, B., Lins, M., \& Pereira, M. (2006). Conhecimento morfossintático e ortografia em crianças do ensino fundamental. Psicologia: Teoria e Pesquisa, 22(1), 9599.

Rego, L. L. B. (1995). Diferenças individuais na aprendizagem inicial da leitura: Papel desempenhado por fatores metalinguísticos. Psicologia: Teoria e Pesquisa, $11(1), 51-60$.

Rego, L. L. B., \& Bryant, P. (1993). The connection between phonological, syntactic and semantic skills and children's reading and spelling. European Journal of Psychology of Education, 8(3), 235-246.

Ryder, J. F., Tunmer, W. E., \& Greaney, K. T. (2008). Explicit instruction in phonemic awareness and phonemically based decoding skills as an intervention strategy for struggling readers in whole language classrooms. Reading and Writing: An Interdisciplinary Journal, 21(4), 349-369.
Santos, A. A. A. (1996). A influência da consciência fonológica na aquisição da leitura e da escrita. In F. F. Sisto, G. C. Oliveira, L. D. T. Fini, M. T. C. C. Souza, \& R. P. Brenelli (Orgs.), Atuação psicopedagógica e aprendizagem escolar (pp. 213-247). Petrópolis, RJ: Vozes.

Shankweiler, D., \& Fowler, A. E. (2004). Questions people ask about the role of phonological processes in learning to read. Reading and Writing: An Interdisciplinary Journal, 17(5), 483-515.

Smith, F. (1971). Understanding reading. New York: Holt, Rinehart \& Winston.

Stanovich, K. (1980). Towards an interactive compensatory model of individual differences in the development of reading fluency. Reading Research Quarterly, 16(1), 33-71.

Sterling, C. (1992). Introduction to the psychology of spelling. In C. Sterling \& C. Robson (Eds.), Psychology, spelling and education (pp. 1-15). Adelaide: Multilingual Matters.

Tunmer, W. T. (1990). The role of language prediction skills in beginning reading. New Zealand Journal of Educational Studies, 25(2), 95-112.

Tunmer, W. T. (1992). Phonological processing and reading recovery: A reply to Clay. New Zealand Journal of Educational Studies, 27(2), 203-217.

Márcia Maria Peruzzi Elia da Mota é Professora Titular do Programa de Pós-graduação em Psicologia da Universidade Salgado de Oliveira e Professora Adjunta da Universidade do Estado do Rio de Janeiro.

Silvia Brilhante Guimarães é Mestre em Psicologia pelo Programa de Pós-graduação em Psicologia da Universidade Federal de Juiz de Fora.
Recebido: 08/09/2009

$1^{a}$ revisão: 20/04/2010

Aceite final: 15/02/2011 